\title{
Characterization of a 2-D Laser Scanner for Mobile Robot Obstacle Negotiation
}

\author{
By \\ Cang Ye and Johann Borenstein* \\ The University of Michigan \\ yecang@engin.umich.edu and johannb@umich.edu* (*Corresponding author)
}

\begin{abstract}
This paper presents a characterization study of the Sick LMS 200 laser scanner. A number of parameters, such as operation time, data transfer rate, target surface properties, as well as the incidence angle, which may potentially affect the sensing performance, are investigated. A probabilistic range measurement model is built based on the experimental results. The paper also analyzes the mixed pixels problem of the scanner.
\end{abstract}

\section{INTRODUCTION}

Range_sensing is a crucial element of any obstacle negotiation system for mobile robots. Sensors suitable for obstacle negotiation are 2-D LADAR (i.e., a laser that scans in one plane) 3-D LADAR (i.e., a laser that scans and "nods," thus producing a range image of an area); Flash LADAR (i.e., a laser that has no moving parts but produces a range image of an area); stereo vision; and millimeter wave radar. An excellent discussion on the state-of-the-art with these sensors is presented in [1].

Given the current state-of-the art we believe that a 3-D laser scanner would be the best choice for building 3-D maps. However, 3-D scanners are inhibitively expensive for most mobile robot applications and many are not fast enough for map building in real time on a fast-moving vehicle, due to the relatively slow vertical scan.

A cost-effective alternative for 3-D mapping is to mount a 2-D laser scanner that is aimed forward and downward on the front end of a mobile robot. During motion the fanning beam of the scanner sweeps over the terrain in front of the robot, effectively creating a 3-D map. This approach is similar to our earlier developed Histogramic In-Motion Mapping algorithm [2], and we believe that a 3-D map with sufficient resolution for obstacle negotiation can be produced this way.

2-D laser scanners are widely used within the mobile robotics community and have been applied to object fol-

\footnotetext{
${ }^{1}$ Obstacle negotiation is the capability of a mobile robot to decide whether an obstacle should be traversed or circumnavigated, and to execute the appropriate action. Obstacle negotiation is typically required in outdoor travel over rugged terrain.
}

lowing and obstacle avoidance [3], feature extraction [4], map building [5,6,7,8] and self-localization [月]. Among the commercially available 2-D laser scanners we found that the LMS 200 made by [Sick] (see Figure 1] matched our obstacle negotiation task best.

Despite their popularity, however, we found that the literature offers only few studies on the performance characteristics of laser scanners. Hoffman and Krotkov [10] used a Perception 3-D laser scanner for terrain map building and studied the influence of target properties and temperature. Langer et al. [1] ] applied a 3-D laser scanner (made by Zoller + Fröhlich [12]) bo railway tunnel surveying and investigated the basic characteristics Figure 1: The Sick LMS 200 (range error and range laser scanner. noise) of the scanner.

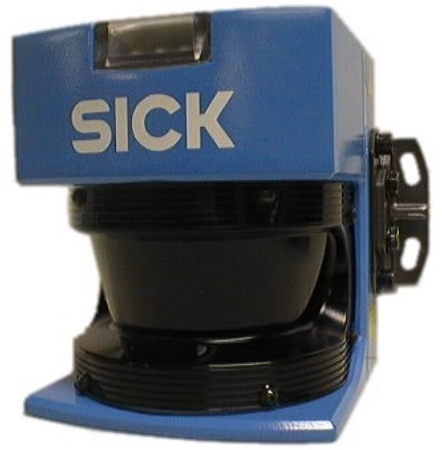
Adams [13] presented his calibration work with a laser scanner where the range error and variance, as well as the distribution of the range measurements for targets with different color, were illustrated.

Adams and Kerstens [13], Hebert and Krotkov [14, and Nitzan [15] all discussed the physics of noise modeling for laser scanners. However, their work was based on 3-D laser scanners using Amplitude Modulated Continuous Wave (AMCW). The AMCW noise model is not applicable to a Time of Flight (TOF) laser scanner like the Sick used in our work. Reina and Gonzalez [16] characterized a TOF laser scanner called "Explorer" (a 2-D scanner made by Schwartz Electro-Optics Inc. [17]) and built a measurement model for that scanner.

This paper presents the results of a characterization study of the Sick LMS 200. We focus specifically on various operational parameters that are relevant to 3-D map building. To the best of our knowledge, no previous work regarding this scanner has been reported in the literature. 
This paper is organized as follows: In Section 2 we present a short functional overview and some technical specifications for the LMS 200 and in Section 3 the experimental setup for our characteristics study is explained. Section 4 investigates various characteristics of the laser scanner and in Section 5 we present a range measurement model derived from the experimental characterization results.

\section{THE SICK LMS 200 LASER SCANNER}

The Sick LMS 200 is a laser scanner based on the measurement of time-of-flight (TOF). As depicted in Figure 2, a pulsed infrared laser beam is emitted and reflected from the object surface. The time between the transmission and the reception of the laser beam is used to measure the distance between the scanner and the object. The laser beam is deflected by a rotating mirror turning at $4500 \mathrm{rpm}$ (75 rps), which results in a fan-shaped scan pattern.

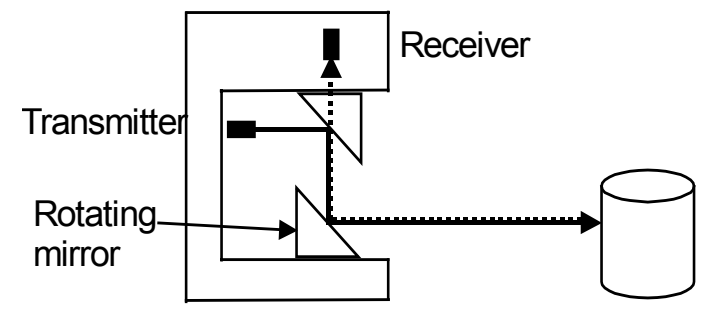

Figure 2: Operating principle of the LMS 200

The angular resolution of the scanner is selectable at $1^{\circ}, 0.5^{\circ}$, or $0.25^{\circ}$. However, the $0.5^{\circ}$ and $0.25^{\circ}$ resolutions are achieved only by interlaced scanning, i.e., a complete frame of data is acquired by 2 or 4 interlaced scans, respectively. For each individual scan, the physical resolution is $1^{\circ}$. We limited our interest to the $1^{\circ}$ resolution setting, where a full scan of $180^{\circ}$ produces 181 measured range values. The data transfer rate can be programmed to be 9.6, 19.2, 38.4, or 500 Kbaud. We used the 500 Kbaud rate, at which data of a full scan is transferred to the user's computer within 13.3 ms. A high-speed RS422 interface card is needed for this transfer rate (unfortunately Sick's support for this high baud rate is very limited and caused us substantial problems). According to the manufacturer's specifications the scanner can measure ranges up to $8 \mathrm{~m}$ with $\pm 15 \mathrm{~mm}$ system error and $5 \mathrm{~mm}$ standard deviation.

\section{EXPERIMENTAL SETUP}

The experiment setup for our characterization and calibration task is depicted in Figure 3. A computer-controlled motor-driven 4-meter linear motion table is used to precisely position a target at specific distances from the scanner. The experiment was carried out in an indoor environment at a temperature of $24^{\circ} \mathrm{C}$. The software used

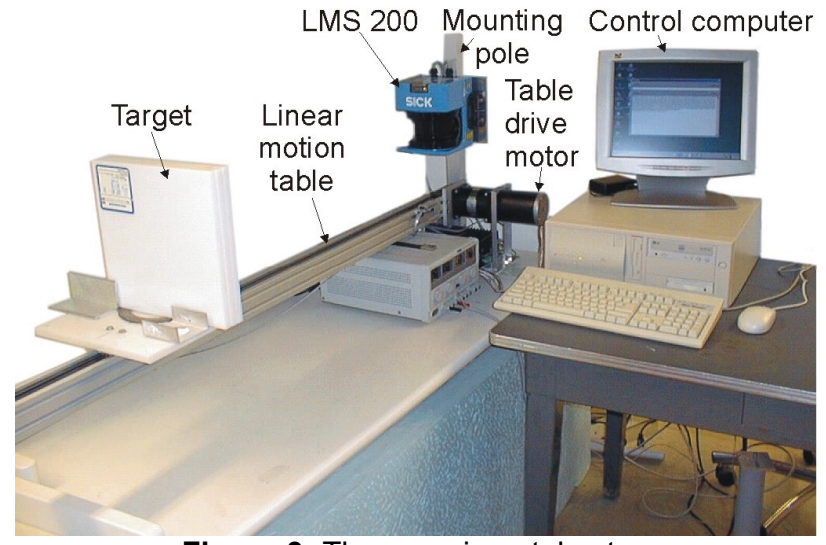

Figure 3: The experimental setup

for the experiment is Visual $\mathrm{C}++6.0$ and the operating system is Window98 SE.

When the scanner was initially mounted on the testbed its orientation (pan and tilt) had to be precisely aligned with the motion table. That is, if aligned properly beam \#91 (throughout this paper we will refer to the 181 range measurements spaced at $1^{\circ}$ increments in a scan as 181 numbered "beams") is perfectly parallel to the longitudinal axis of the motion table. To do so we performed the following calibration procedure:

1. Place the target at a distance of $330 \mathrm{~mm}$ from the mounting pole and read the measured range $d_{m 1}$ (actually, we averaged 10,000 samples of the $91^{\text {st }}$ value) from the scanner.

2. Move the target $3,800 \mathrm{~mm}$ forward and read the measured range $d_{m 2}$.

3. If $d_{m 2^{2}} d_{m l}$ is larger than $3,800 \mathrm{~mm}$, change the tilt angle of the scanner and repeat steps 1 . and 2. until $d_{m 2}-d_{m 1}$ reaches a minimum value.

4. Identify the beam number at which $d_{m 2}-d_{m 1}$ reaches a new minimum value. Following the above procedure, we found that the $89^{\text {th }}$ beam (i.e., the $89^{\text {th }}$ measured value of a scan) produced a minimum value of $3,802.2$ $\mathrm{mm}$ for $d_{m 2}-d_{m 1}$. Therefore, we could assume that the $89^{\text {th }}$ beam was the beam that was most closely aligned with the longitudinal axis of the motion table and that beam was subsequently used in our experiments.

\section{ChARACTERISTICS OF THE LASER SCANNER}

In this section we present a number of experiments aiming at characterizing the laser scanner. In particular, we analyze the effect of various operating parameters relevant to map building. They are the data transfer rate, drift, the optical properties of the target surfaces, and the incidence angle of the laser beam.

\subsection{Effect of the data transfer rate}

For fast moving mobile robots it is important to use the fastest data transfer rate available with the Sick LM 200, $500 \mathrm{Kbaud}$. Using a transfer protocol, in which the scan- 
ner sends a continuous data stream and using the $1^{\circ}$ angular resolution, a data packet for a complete scan is 372 bytes long, beginning with a 7-byte header and ending with a single 1-byte status and a 2-byte checksum. The time overhead for transferring a data packet is $7.4 \mathrm{~ms}$ while the time to complete a full scan is $13.3 \mathrm{~ms}$.

In our system we found that occasionally data packets were lost when communication took place at $500 \mathrm{Kbaud}$, while no packets were lost at the much slower $38 \mathrm{Kbaud}$ rate. We tested this condition extensively and found that the number of lost data packets never exceeded $0.08 \%$ of all data packets sent. We developed at work-around that allows us to detect and discard incomplete packets. This way lost data packets don't introduce false readings and we concluded that the problem was negligible and that the system was fully usable at 500 Kbaud.

\subsection{Effect of Drift}

In this experiment, the target was placed 2 meters away from the scanner. We then sampled 600,000 complete scans and the mean of every 1,000 samples was taken as the measured range. Figure 4 depicts the drift of the measured range over the duration of this experiment. One can observe fairly large variations for the first 10,000 seconds ( 2 hours and 46 minutes). However, the measured range is more stable after 10,000 seconds. To reduce the effect of drift on other characterization parameters, all data presented in this paper was acquired after an extended warm-up time of about 3 hours.

\subsection{Effect of target surface properties}

In order to characterize the LMS 200 scanner with regard to different reflectance properties, we ran tests with 13 different targets organized in three groups. The target surface categories were ${ }^{2}$.

Shiny colors: Cardboard with gold-colored, reflective surface, cardboard with silver-colored reflective surface, and a sheet of polished aluminum.

Matted colors: Five cardboards with colored surfaces: blue, purple, red, green, and yellow.

Gray levels: Standard white copier paper that was entirely covered with laser printer-produced gray-level output: $\mathrm{RGB}=255, \mathrm{RGB}=191, \mathrm{RGB}=127, \mathrm{RGB}=63$, and $\mathrm{RGB}=0$.

The targets were initially placed $192 \mathrm{~mm}$ in front of the scanner. During the experiment the motion table moved the targets further away, in 500-millimeter increments, up to a distance of 3,692 $\mathrm{mm}$. At each incremental position 10,000 samples were taken and the distribution of the measured range values was evaluated.

\footnotetext{
${ }^{2}$ Vendor: http://www.daler- rowney.co.uk/cat/usa/index.html Product: Canford Cardboards. Vendor's names for colors: "Frosted Gold," "Frosted Silver;" "Bright Red," "Royal Blue," "Dresden Yellow," "Emerald Green," "Amethyst" (= "purple" in this paper).
}

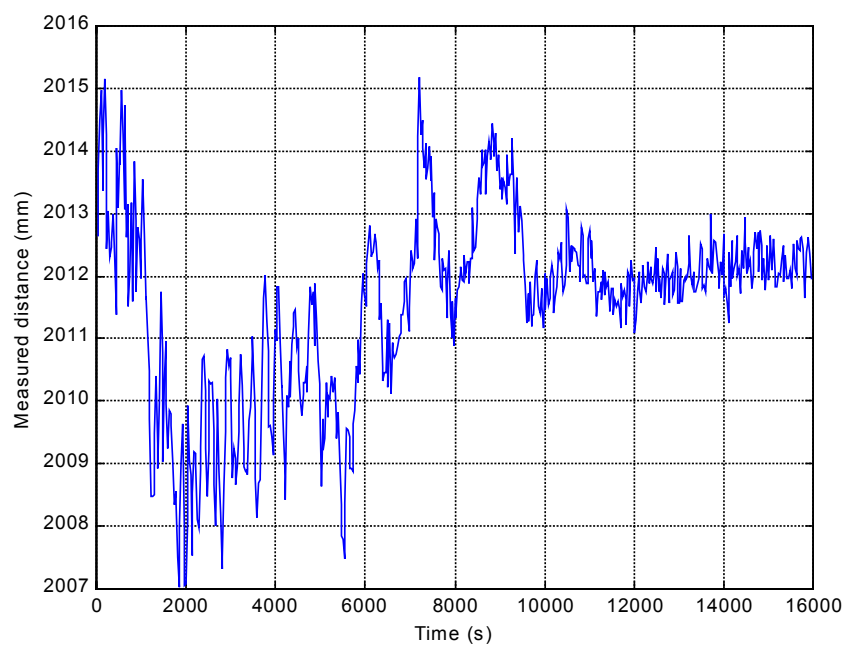

Figure 4: Drift of range over time: Range fluctuations up to $8 \mathrm{~mm}$ can be observed for the first 3 hours. After 3 hours the range fluctuations decrease to $2 \mathrm{~mm}$.

As depicted in Figure 5a, the measured range values for the shiny targets have approximate normal distributions. Targets with stronger reflectivity (reflectivity scale: aluminum $>$ silver $>$ gold) have a narrower spread and a higher peak. The plots of the data at various distances show that a wider spread and a lower peak is produced for larger distances. This is due to the fact that the intensity of the reflected light from a farther target is smaller, thus resulting in a larger variance. The maximum standard deviations for the full range $(192-3,692 \mathrm{~mm})$ are $\sigma_{\max }=4.0, \sigma_{\max }=3.5$, and $\sigma_{\max }=2.1$ for the gold, silver, and aluminum targets, respectively. The mean values do not change significantly.

The distributions of the measured range values for the color targets consist of two lobes, each of which approximately a normal distribution (Figure 5 ). The heights of the lobes may vary with the distance and at some points the smaller lobe may disappear. The width of the spread and the height of the peak are similar to those for the shiny targets, but they do not vary significantly. The maximum standard deviations for the full range are $\sigma_{\max }=4.3, \sigma_{\max }=4.0, \sigma_{\max }=3.9, \sigma_{\max }=3.8$ and $\sigma_{\max }=3.4$ for the red, purple, yellow, blue, and green target, respectively. The mean values do not change notably either. We thus conclude that target surface color does not affect range measurement significantly.

For the targets with gray levels, the distributions of the measured range values are composed of a number of approximate normal distributions, i.e., a higher main lobe with a number of lower side lobes (see Figure 54). Similarly, the heights of the lobes vary with the distances, and the number of side lobes differ. Again the widths of the spreads and the heights of the peaks are similar to those for the colored and shiny targets. The standard deviations are $\sigma_{\max }=5.5, \sigma_{\max }=6.5, \sigma_{\max }=5.5, \sigma_{\max }=4.7$, and $\sigma_{\max }=3.0$ for $\mathrm{RGB}=0,63,127,191$, and 255 , respectively. The de- 

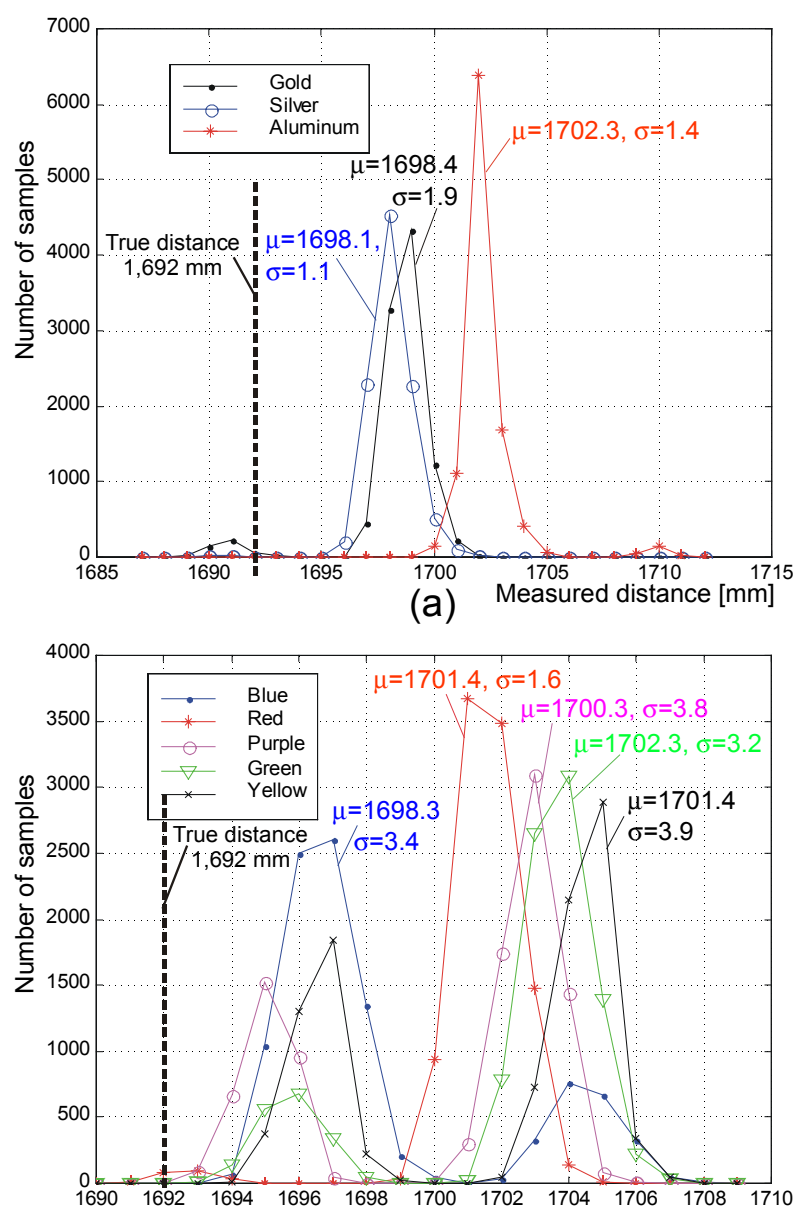

(b) Measured distance [mm]

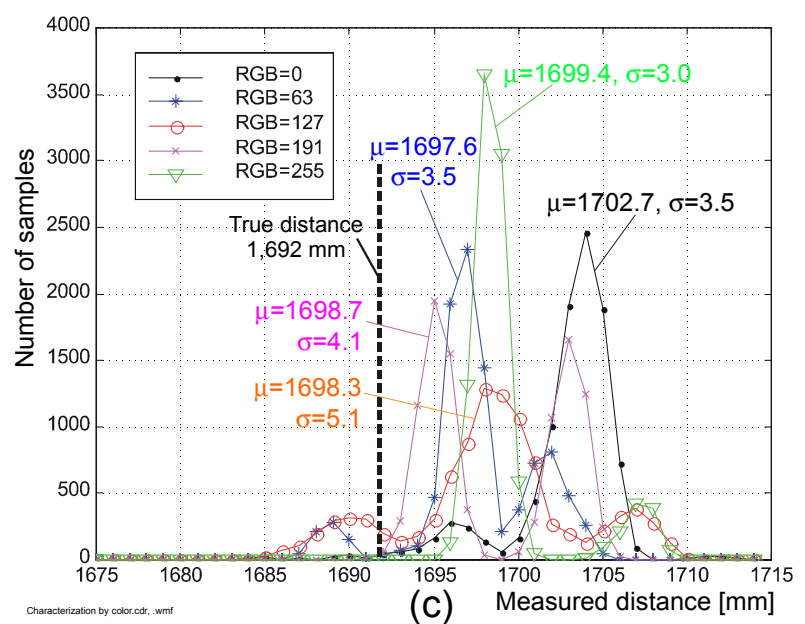

Figure 5: Distribution of measured range. Shown here are the distributions for a true distance of 1,692 $\mathrm{mm}$. (a) Shiny materials, (b) matted color cardboards, (c) matted gray paper.

viations are relatively larger. Compared with the results for the color targets, the variations of the mean values due to different gray levels are larger. This means that the gray level of a target has a more significant impact on the distribution of the range measurement.
In summary, the target surface properties do not have significant influence on the mean of the measured ranges but they affect the distributions of the measured ranges significantly. One exception is that the color properties have only a minor effect on distributions.

\subsection{Effect of the incidence angle}

In this section we examine the effect that different incident angles have on the range measurement accuracy. In the proceeding section this angle was constant at $0^{\circ}$.

One potential problem when conducting experiments with an incident angle other than $0^{\circ}$ is that the true distance between the scanner and the target is now harder to determine. This problem is illustrated in Figure 6. if both the scanner and the rotation axis of the target were located on the longitudinal center line of the linear table ( $a$ and $b$, respectively), then the true distance between the scanner and the target would always be $\overline{a b}$ regardless of the target's orientation. However, due to measurement errors during installation, we must assume that the scanner and the rotation axis of the target deviated by small distances ( $q$ and $p$, respectively, in Figure 6 from the linear motion table's longitudinal centerline. This introduces an additional error $\pm(p+q) \operatorname{tg} \theta$ (i.e., the true distance is $\overline{a b} \pm(p+q) \operatorname{tg} \theta)$ if the target rotates an angle of $\theta$ clockwise or counter-clockwise. Since this error is not caused by an optical effect of the incidence angle or by the scanner it must be eliminated before measuring scanner errors. One way to eliminate this error is by computing the average of the pair of range values measured with target orientations at $\theta$ and $-\theta$.

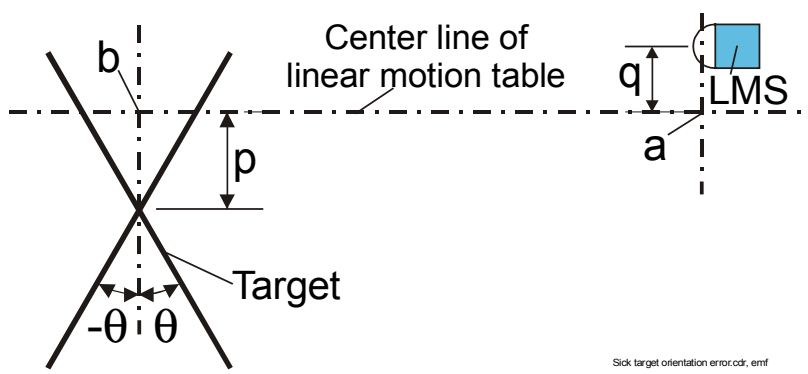

Figure 6: Additional error introduced by object orientation

For this experiment, the gray target with $\mathrm{RGB}=127$ was positioned 2 meters in front of the scanner and 13 different orientation angles were set up using a protractor. These angles were: $0^{\circ}, \pm 10^{\circ}, \pm 20^{\circ}, \pm 30^{\circ}, \pm 40^{\circ}, \pm 50^{\circ}$, and $\pm 60^{\circ}$ (we were not able to get reliable range measurements for $\theta \geq 70^{\circ}$ ). For each angle, 10,000 samples were taken. Then the data pairs (e.g., data sampled at the positive and negative orientation) were averaged and the means of the measured range values were computed for $\pm 10^{\circ}, \pm 20^{\circ}, \pm 30^{\circ}, \pm 40^{\circ}, \pm 50^{\circ}$ and $\pm 60^{\circ}$, respectively. Figure 7 depicts the distributions of the measured ranges for the target at different orientations. 


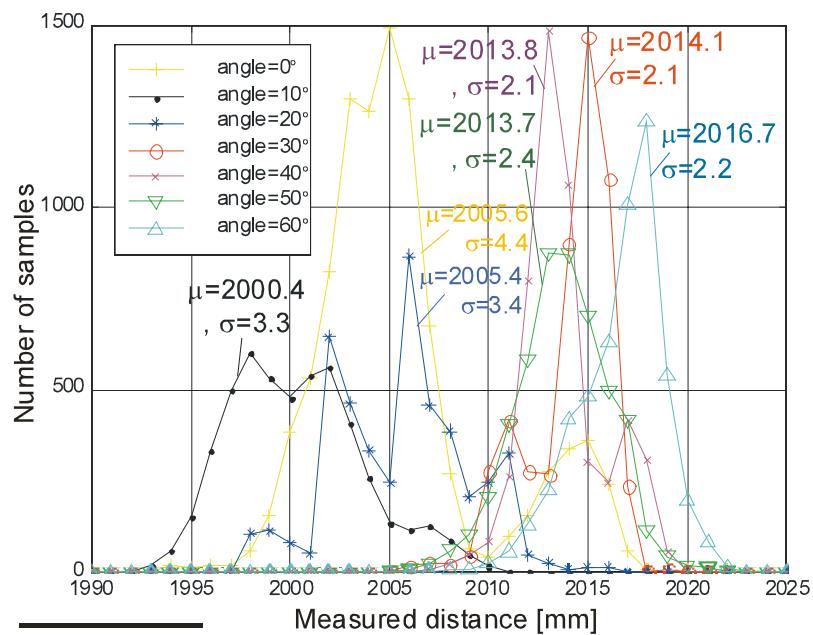

Figure 7: Distribution of ranges at various orientations.

One can observe that the height of the peak for each data set is low, which agrees with Figure 5t where the distribution curve of $\mathrm{RGB}=127$ has a lower peak. The curves and mean values for $\pm 0^{\circ}, \pm 10^{\circ}$, and $\pm 20^{\circ}$ are close to each other suggesting that an incidence angle between $-20^{\circ}$ and $+20^{\circ}$ will not cause a significant error in the range measurements. A relatively larger error was caused by the incidence angle of $30^{\circ}$ and the error is small again from $30^{\circ}-60^{\circ}$. The difference between the largest (mean) distance measured and the smallest (mean) distance measured (at incidence angles of $\pm 60^{\circ}$ and $\pm 10^{\circ}$, respectively) was about $16 \mathrm{~mm}$. This suggests that the incidence angle has a greater influence than the target gray level on the mean of the range measurement.

\subsection{The mixed pixels problem}

When a laser spot is located at the very edge of an object, the measured range is that of a combination of the foreground object and the background object, i.e., the range falls in between the distances to the foreground and background objects. This condition is called "mixed pixels" [13. To investigate this condition, we positioned a target at 1 meter, and a uniform background at about 2 meters in front of the scanner. At this distance 41 beams (from beam no. 71 to 111) hit the target, while all other beams hit the background.

Figure 8 shows a plot of the mean from 250 scans in Cartesian coordinates. Two mixed pixels, labeled $\mathrm{a}_{1}$ and $\mathrm{a}_{2}$, were found between 1 meter and 2 meters. As one might expect, if the target was slightly moved to the left, then the range reading associated with $\mathrm{a}_{2}$ moved closer to the background object while the range reading associated with $a_{1}$ moved closer to the target. One should note that the mixed pixels phenomenon exists even in a single scan, that is, it is not the result of averaging values of multiple scans.

The mixed pixels condition may also be found when the laser spot is located at an area where the reflectivity of

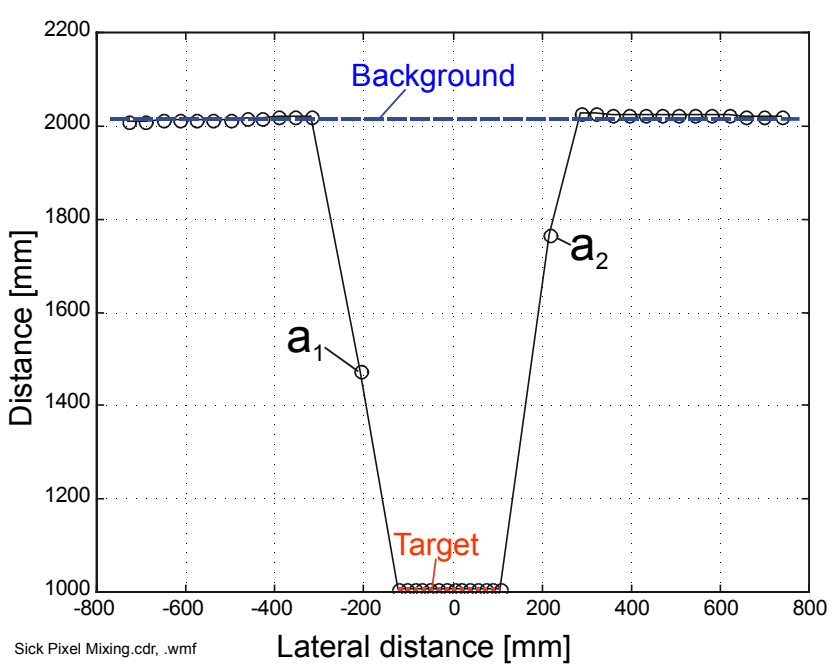

Figure 8: So-called mixed pixels $\mathrm{a}_{1}$ and $\mathrm{a}_{2}$ can occur at the edges of a target. As a result, mean distances $a_{1}$ and $a_{2}$ are incorrectly located somewhere between the target and the background.

the surface changes sharply. However, from Figure 5 it can be inferred that the induced measurement error is small enough to be ignored in that case.

\section{CALIBRATION AND SimULATON MODELS}

Based on our empirical data we built a mathematical model of the scanner errors. This model can be used as a calibration function to reduce measurement errors.

\subsection{The calibration model}

To calibrate the measured range values, the targets were placed at a position from $y_{1}=212 \mathrm{~mm}$ to $y_{192}=4012 \mathrm{~mm}$ in increments of $20 \mathrm{~mm}$. At each position $y_{i}, 4,000$ samples, $z_{j i}$ for $j=1, \ldots, 4,000$, were taken and the mean, denoted as $\mu_{i}$, was calculated. The 13 targets introduced in Section 4 were used to collect the data. We found that the mean had an approximate linear relationship with the true distance for each target. Therefore, the true distance may be estimated by a linear function as follows:

$$
\hat{y}=k \mu+b
$$

where $\hat{y}$ is the estimate of the true distance $y$ and $\mu$ is the mean of measured range. $k$ and $b$ are constant and their values should minimize the square error given by

$$
\sum_{i=1}^{n}\left(y_{i}-\hat{y}_{i}\right)^{2}=\sum_{i=1}^{n}\left(y_{i}-k \mu_{i}-b\right)^{2}
$$

Therefore, we have

$$
\left\{\begin{array}{l}
k=\frac{\sum_{i=1}^{n}\left(y_{i}-\bar{y}\right)\left(\mu_{i}-\bar{u}\right)}{\sum_{i=1}^{n}\left(u_{i}-\bar{u}\right)^{2}} \\
b=\bar{y}-k \bar{\mu}
\end{array}\right.
$$


where $\bar{y}$ and $\bar{\mu}$ are the mean values of $y_{i}$ and $\mu_{i}$, respectively.

Ideally, the calibration should account for the orientation and the surface properties of the targets. However, surface properties are not usually known in most mobile robot obstacle negotiation applications. Therefore, for our application we use only the data acquired for a medium gray target $(\mathrm{RGB}=127)$ at $0^{\circ}$ incidence angle to calibrate the measured ranges. With this data $k=1.0002$ and $b=-3.6$.

Eq. (1) can be used as a calibration function that corrects range measurements for empirically determined errors of the scanner. We use this function to calibrate the measured ranges of the 13 targets and the result is depicted in Figure 9 where Figure 9 depicts the range error $\hat{y}-y$ vs. the true distance $\mathrm{y}$ and Figure 93 depicts the standard deviations. The range errors for the 13 different targets have an upper bound of $14.5 \mathrm{~mm}$ and a lower bound of $-16.9 \mathrm{~mm}$ while the standard deviations are bounded by $(0.8,9.1)$. These values are larger than the specifications provided by the manufacturer.

\subsection{The range measurement model}

The range measurement model is a simulation model, which estimates the measured range using the true distance. It may be used to simulate the scanner function in simulations.

Since the mean of the measured range has an approximately linear relationship with the true distance, it may be approximated by a linear function:

$$
\hat{\mu}=n y+c
$$

where $\hat{\mu}$ is the estimate of $\mu$ and $y$ is the true distance; while $n$ and $c$ are constant. Following the same method as in the preceding section, we have

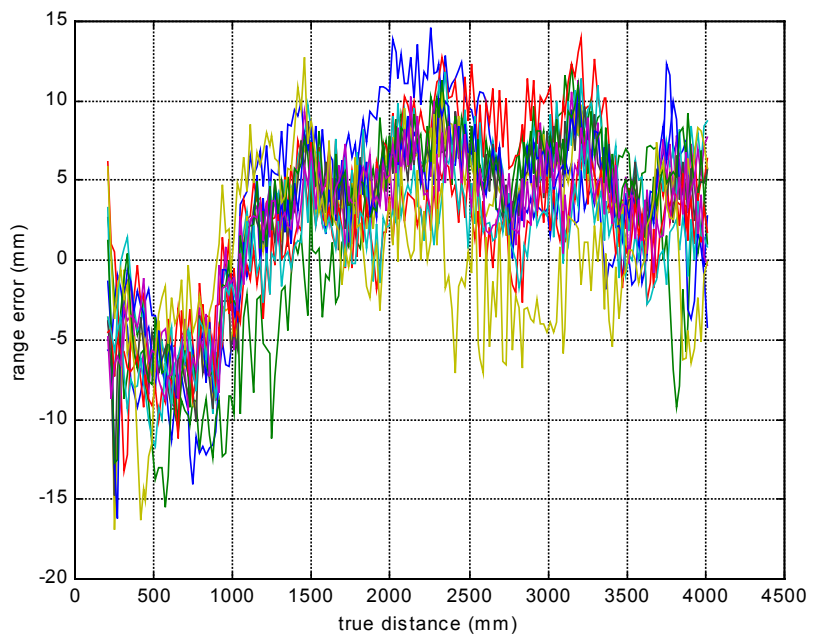

(a)

$$
\left\{\begin{array}{l}
n=\frac{\sum_{i=1}^{n}\left(y_{i}-\bar{y}\right)\left(\mu_{i}-\bar{u}\right)}{\sum_{i=1}^{n}\left(y_{i}-\bar{y}\right)^{2}} \\
c=\bar{\mu}-n \bar{y}
\end{array}\right.
$$

Assuming the measurement error is Gaussian noise, the simulated measured range value of a single scan is

$$
z_{s}=\operatorname{Int}(n y+c+\xi)
$$

where $\xi$ is Gaussian noise with a normal distribution $N(0, \sigma)$. The rounding function Int(.) is applied to the measurement model as the physical scanner produces integer range values.

We also used the data acquired by a medium gray target $(\mathrm{RGB}=127)$ at $0^{\circ}$ incidence angle to model the range measurements. With this data set, $n=0.9998$ and $c=3.6$.

The value of $\sigma$ in Eq. (6) determines how closely the simulation model matches the physical scanner. We used the calibrated range measurements of the target at distances from $1,992 \mathrm{~mm}$ to $2,592 \mathrm{~mm}$. In this range, the minimum and maximum standard deviation are 2.7 and 7.0 , respectively. The process for determining the value of $\sigma$ is as follows:

1. The distribution of the calibrated range measurements (measured by the physical scanner and calibrated by Eq. (1)) for each distance from 1,992 mm to $2,592 \mathrm{~mm}$ was calculated.

2. The simulated range measurement was computed by Eq. (6) where $y$ assumes value from $1,992 \mathrm{~mm}$ to $2,592 \mathrm{~mm}$ in increments of $20 \mathrm{~mm}$ and $\sigma$ from 2.7 to 7.0 in increments of 0.1 . For each position and $\sigma$ value, 4,000 simulated range values were calculated and the distribution was computed.

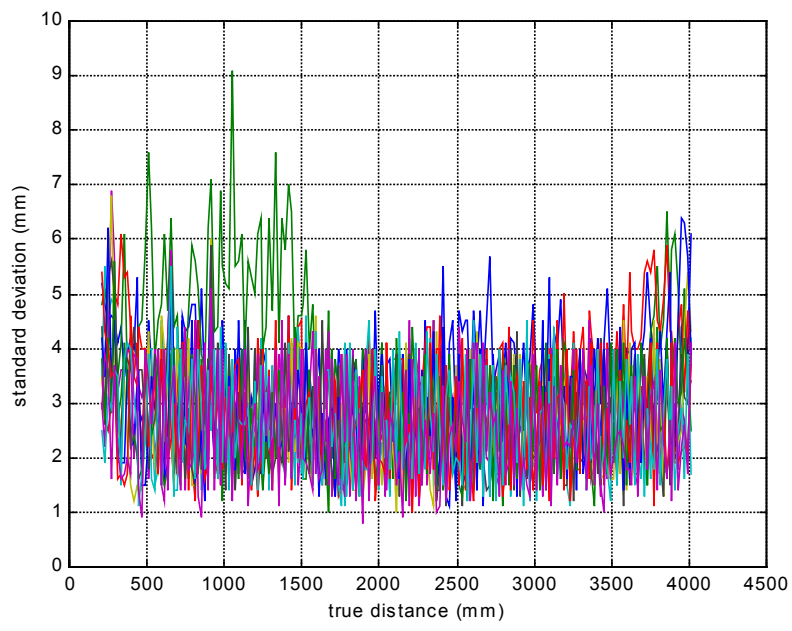

(b)

Figure 9: Measurement error after calibration for the 13 targets. (a) Range error vs. true distance; (b) standard deviation vs. true distance. 
3. The mean square error between these two distributions was calculated for each $\sigma$ by

$$
\frac{1}{31} \sum_{i=1}^{31} \sum_{j=-2}^{2}\left(N S_{i}(j)-N R_{i}(j)\right)^{2}
$$

where $N S_{i}$ and $N R_{i}$ are the number of samples for the simulated and real distributions, respectively. The parameter $i$ is the index of the position from 1,992 $\mathrm{mm}$ to 2592 while $j$ is the index of the five most significant numbers of samples. The mean square error achieved its minimum at $\sigma=5.3$. Therefore, $\sigma=5.3$ is used to model the Gaussian noise for Eq. (6).

After the values of $n, c$ and $\sigma$ were determined, the model (Eq. (6)) was validated with other sets of range measurements. In all cases the mean square error computed by Eq. (7) was sufficiently small to validate our approximation assuming Gaussian noise.

\section{Conclusions}

In this paper, the Sick LMS 200 laser scanner was characterized through extensive experimentation. One problem of the scanner, unrelated to characterization, is that it may encounter loss of synchronization at its highest data transfer rate. We identified this problem and introduced a workaround.

Our experiments suggest that there is noticeable drift up to 3 hours after start-up, but that drift-related fluctuations stabilize after that time. We also found that - similar to other laser scanners - so-called mixed pixels may occur in range measurements.

Other measurement errors are caused by target surface reflectance properties and the incidence angle of the laser beam. Maximal errors for most cases were on the order of $17 \mathrm{~mm}$. We believe that these errors are quite acceptable for in-motion map building on mobile robots, since possible errors induced by the positioning system and other application-related effects could easily be one order of magnitude larger.

The range measurement model for the LMS 200 introduced in this paper may be used for testing algorithms in simulation.

\section{Acknowledgements}

This work was funded by the U.S. Department of Energy under Award No. DE-FG04-86NE3796.

\section{REFERENCES}

[1] M. Hebert, "Active and passive range sensing for robotics", Proc. Int. Conf. on Robotics and Automation, San Francisco, CA, April, pp. 102-110, 2000.

[2] J. Borenstein and Y. Koren, "Histogramic in-motion mapping for mobile robot obstacle avoidance", IEEE Trans. on Robotics and Automation, Vol. 7, No. 4, pp.535-539, 1991.
[3] J. L. Martínez, et al, "Object following and obstacle avoidance using a laser scanner in outdoor mobile robot auriga- $\alpha$ ", Proc. of IEEE/RSJ Int. Conf. on Intelligent Robots and Systems, pp. 204-209, 1998.

[4] S. I. Roumeliotis and G. A. Bekey, "SEGEMENTS: A layered, dual-Kalman filter algorithm for indoor feature extraction", Proc. of IEEE/RSJ Int. Conf. on Intelligent Robots and Systems, pp. 454-461, 2000.

[5] Y. D. Kwon and J. S. Lee, "A stochastic map building method for mobile robot using 2-D laser range finder", Autonomous Robots, Vol. 7, No.2, pp. 187200, 1999.

[6] B. Giesler, et al, "Fast mapping using the Log-Hough Transformation", Proc. IEEE/RSJ Int. Conf. on Intelligent Robots and Systems, pp. 1702-1707, 1998.

[7] A. Scott, et al, "Quantitative and qualitative comparison of three laser-range mapping algorithms using two types of laser scanner data", Proc. IEEE Int. Conf. on Systems, Man, and Cybernetics, pp. 14221427, 2000.

[8] J. Guivant, et al, "High accuracy navigation using laser range sensors in outdoor application", Proc. IEEE Int. Conf. on Robotics and Automation, pp. 3817-3821, 2000.

[9] L. Zhang and B. K. Ghosh, "Line segment based map building and localization using 2D laser rangefinder", Proc. IEEE Int. Conf. on Robotics and Automation, pp. 2538-2543, 2000.

[10]R. Hoffman and E. Krotkov, "Perception of rugged terrain for a walking robot: true confession and new directions", Proc. of IEEE/RSJ Int. Conf. on Intelligent Robots and Systems, pp. 1505-1510, 1991.

[11]D. Langer, et al, "Imaging laser scanner for 3-D modeling and surveying applications", Proc. IEEE Int. Conf. on Robotics and Automation, pp. 116-121, 2000.

[12] http://www.zofre.de/eallg.htm

[13] M.D. Adams and A.J. Kerstens, "The interpretation of phase and intensity data from AMCW light detection sensors for reliable ranging", Int. J. of Robotics Research, Vol. 15, No.5, pp. 441-458, 1996.

[14] M. Hebert and E. Krotkov, "3D measurements from imaging laser radars: how good are they?", Image and Vision Computing, vol. 10, no. 3, pp. 170$178,1992$.

[15]D. Nitzan, et al, "The measurement and use of registered reflectance and range data in scene analysis", Proceedings of the IEEE, vol. 65, no. 2, 1977.

[16] Reina and J. Gozalez, "Characterization of a radial laser scanner for mobile robotic navigation,"Proc. IEEE/RSJ Int. Conf. on Intelligent robots and systems, pp. 579-585, 1997.

[17] http://www.seord.com/ 